\section{Consumo de álcool e drogas ilícitas e envolvimento de adolescentes em violência física em Pernambuco, Brasil}

\author{
Alcohol and illicit drug consumption and \\ involvement by adolescents in physical \\ violence in Pernambuco, Brazil
}

\section{Consumo de alcohol y drogas ilícitas e implicación de adolescentes en violencia física en Pernambuco, Brasil}

Daniel da Rocha Queiroz ${ }^{1}$ Mauro Virgílio Gomes de Barros 1 Javiera Alarcón Aguilar 1 Fernanda Cunha Soares 1 Rafael de Miranda Tassitano 2 Jorge Bezerra 1 Lygia Maria Pereira da Silva 1

\section{Resumo}

O objetivo foi estimar a prevalência e analisar a associação do consumo de álcool e drogas ilícitas com indicadores de violência física em adolescentes. Estudo transversal repetido com estudantes do Ensino Médio em escolas da rede pública em Pernambuco, Brasil (2006, $n=4.207$; 2011, $n=6.264$ ). Os dados foram obtidos por questionário. Os indicadores de violência física foram: vitimização por violência física; envolvimento em brigas. As variáveis independentes foram: consumo de álcool e uso de drogas ilícitas. A regressão logística binária foi utilizada para verificar a associação entre o consumo de álcool e drogas e ter sofrido violência física. A prevalência do consumo de álcool por adolescentes que sofreram violência física reduziu em cinco anos, porém, nenhuma alteração foi constatada no uso de drogas ilícitas. As variáveis comportamentais estiveram associadas aos diferentes indicadores de violência, apresentando em geral uma tendência de aumento. Em 2006, adolescentes que usavam drogas ilicitas apresentaram uma chance 2,12 maior de sofrer violência física, e em 2011 este valor foi de 2,41 (IC95\%: 1,80; 3,22). A chance de adolescentes que consumiam bebida alcoólica ter envolvimento em brigas aumentou de 1,96 (IC95\%: 1,64; 2,34) em 2006, para 2, 11 (IC95\%: 1,78; 2,51) em 2011. O consumo de álcool pelos adolescentes que sofreram violência física diminuiu em cinco anos, entretanto, o risco de os adolescentes que consumiram bebida alcoólica ou usaram drogas ilícitas sofrerem violência física aumentou ao longo de cinco anos.

Consumo de Bebidas Alcoólicas; Drogas Ilícitas; Saúde do Adolescente; Violência

\author{
Correspondência \\ F. C. Soares \\ Av. Agamenon Magalhães s/n, Recife, PE 50100-010, Brasil. \\ fercsoares@gmail.com \\ 1 Universidade de Pernambuco, Recife, Brasil. \\ 2 Universidade Federal Rural de Pernambuco, Recife, Brasil.
}




\section{Introdução}

Violência é caracterizada como um fenômeno complexo e dinâmico com raízes biológicas, psicológicas e sociais, tendo como cenário de origem e desenvolvimento a vida em sociedade 1. Violência, tanto quanto acidentes e enfermidades crônico-degenerativas, divide o cenário epidemiológico mundial como um novo perfil de agravos à saúde 2. Sofrer violência pode causar problemas no comportamento, dificuldades no desenvolvimento e efeitos nocivos na saúde física e mental, podendo perdurar por toda a vida 3. A violência pode ser classificada por sua natureza como física, sexual, psicológica ou privação/ negligência. A violência interpessoal subdivide-se em violência familiar e violência comunitária 4 .

A violência física é apresentada como um tipo de violência comum entre os adolescentes 5 , período de intensas mudanças físicas, psicologias e sociais, que podem modular a transição da infância para a fase adulta 6 . As taxas de violência física atingem o pico durante a adolescência, e os adolescentes sofrem desproporcionalmente as consequências desta violência, incluindo prisão, ferimentos e morte 7 . Entre 2009 e 2010, estima-se que 13\% dos estudantes de 13 anos e 10\% dos estudantes de 15 anos da Europa, América do Norte e Israel estiveram envolvidos em brigas 8. Em 2011, no Brasil, de 100 mil casos de violência física atendidos pelo Sistema Único de Saúde (SUS), 25\% eram de adolescentes entre 10 e 19 anos. No Nordeste, a cada 100 mil atendimentos, 4.776 adolescentes buscaram o serviço de saúde por causa associada à violência física, representando $29,8 \%$ do total de casos na região 9 .

A adolescência também é caracterizada por uma fase de aquisição de condutas de saúde ou de exposição a fatores de risco à saúde 10, e alguns destes fatores de risco parecem estar associados à violência física. Estudos indicam que o uso de álcool e a violência são comportamentos de risco fortemente inter-relacionados que tendem a atingir o pico durante a adolescência 11,12. Jovens que usam qualquer droga ilícita parecem ter duas vezes mais chances de se envolverem em violência em comparação com jovens que não relatam uso de drogas ilícitas 13 .

Apesar de ser uma das principais causas de morte e incapacidade entre jovens de países em desenvolvimento 14, a violência física ainda é um tema que tem recebido pouca atenção no campo da pesquisa nesta população 15,16. Dessa forma, torna-se importante a avaliação de fatores comportamentais associados aos diferentes tipos de violência física em adolescentes em um país em desenvolvimento, tendo em vista a dificuldade de encontrar consenso na literatura sobre as variáveis de análise de risco ou proteção, o número reduzido de variáveis analisadas e a ausência de modelos teóricos explicativos 17. Devido à lacuna existente na associação entre tipos de violência física e uso de álcool e outras drogas por adolescentes brasileiros, o objetivo do presente estudo foi estimar a prevalência e analisar a associação do uso de álcool e outras drogas com violência em adolescentes.

\section{Métodos}

Estudo transversal repetido 18, realizado como parte de um levantamento epidemiológico de base escolar e abrangência estadual, intitulado Práticas de Atividades Físicas e Comportamentos de Risco à Saúde em Estudantes do Ensino Médio no Estado de Pernambuco: Estudo de Tendência Temporal (20062011). Levantamentos transversais foram realizados em 2006 e 2011, e o protocolo de pesquisa foi aprovado pelo Comitê de Ética em Pesquisa com Seres Humanos da Universidade de Pernambuco (56341416.9.0000.5192/CEP-UPE). Todos os pais/responsáveis foram informados do estudo por meio do Termo de Consentimento Livre e Esclarecido e os adolescentes pelo Termo de Assentimento.

A população-alvo foi constituída por estudantes de Ensino Médio matriculados nas escolas da rede pública estadual de Pernambuco, Brasil, com faixa etária entre 14 e 19 anos. Os detalhes sobre o Projeto ATITUDE podem ser acessados no artigo publicado 19, apenas as variáveis de interesse do presente estudo serão detalhadas neste manuscrito. O cálculo amostral dos dois levantamentos considerou um intervalo de $95 \%$ de confiança (IC95\%), um erro máximo tolerável de $2 \%$ e uma prevalência estimada de variáveis de 50\%. Além disso, foi aplicada uma correção (efeito de desenho - deff), considerando que a seleção da amostra ocorreu por conglomerado em duas etapas (escolas e turmas). Em 2006, o deff adotado foi de 4, já o deff em 2011 foi de 2. A amostra mínima foi inflada em 20\% em todos os levantamentos devido a perdas e recusas. Em 2006, 4.207 adolescentes foram monitorados, e 6.264 adolescentes foram incluídos em 2011. 
As informações foram obtidas mediante a utilização de uma versão traduzida, autoadministrada e previamente testada do Global School-Based Student Health Survey (GSHS). Um estudo piloto foi conduzido com o objetivo de determinar indicadores de reprodutibilidade do instrumento, mostrando boa consistência de medidas, validade de conteúdo e de face. No primeiro inquérito, o índice kappa variou entre 0,52-1,00 (concordância de moderada à perfeita), e no segundo variou entre 0,63-0,98 (concordância de forte à perfeita).

A aplicação do questionário foi realizada em sala de aula, sem a presença dos professores, para todos os alunos presentes. Inicialmente foram informados os objetivos da pesquisa, os alunos foram orientados a não se identificarem no questionário, esclarecendo-se aos estudantes que as informações fornecidas seriam mantidas em sigilo, não influenciando no seu desempenho escolar e que só seriam utilizadas para fins de pesquisa. A aplicação do questionário era realizada por meio de entrevista coletiva.

As informações sobre violência física (variáveis dependentes) foram retiradas de duas questões: (a) vitimização por violência física: "Durante os últimos 12 meses, quantas vezes você sofreu algum tipo de violência física?”; (b) envolvimento com brigas: "Durante os últimos 12 meses, quantas vezes você esteve envolvido numa briga?”. As opções de respostas de ambas as questões eram: nenhuma vez; 1 vez; 2 ou 3 vezes; 4 ou 5 vezes; 6 ou 7 vezes; 8 ou 9 vezes; 10 ou 11 vezes; 12 vezes ou mais. As variáveis foram categorizadas em não ter sofrido violência (nenhuma vez) e ter sofrido violência (1 vez ou mais).

As variáveis independentes foram divididas em dois blocos: (1) variáveis sociodemográficas (bloco distal) - sexo (rapazes e moças), turno (diurno e noturno), idade (14-16 e 17-19 anos), escolaridade materna ( $\leq 8,9-11$ e $\geq 12$ anos), local de residência (urbana e rural), cor da pele (branca, preta e outra), série (1o ano, 2o ano e 3o ano); religião (não possui, católico, evangélico e outra), estado civil (solteiro, casado e outro); (2) variáveis comportamentais (bloco proximal) - (a) consumo de álcool durante a vida (não e sim); (b) uso de drogas ilícitas (não legalizadas como maconha, cocaína, êxtase etc) durante a vida (não e sim); (c) consumo de tabaco durante a vida (não e sim). Para fins de análise, as variáveis dependentes e as variáveis independentes comportamentais do bloco proximal foram dicotomizadas (sim e não).

As tabulações foram realizadas utilizando-se o EpiData (http://www.epidata.dk/), por meio de procedimentos eletrônicos de entrada de dados. Foi usada a dupla digitação para identificar a consistência da entrada dos dados e corrigir os possíveis erros de digitação. Para a análise dos dados foi utilizado o pacote estatístico Stata versão 11.2 (https://www.stata.com), nas análises descritivas foi usada a distribuição de frequências, para as análises inferenciais bivariadas, o teste de qui-quadrado e o valor da variação das estimativas pelo delta percentual $(\Delta \%)$.

$\mathrm{Na}$ análise multivariada foi usada a regressão logística binária. Um modelo hierárquico, com base no modelo ecológico do desenvolvimento, proposto por Bronfenbrenner 20, foi adotado para a entrada das variáveis independentes, utilizando-se um modelo conceitual com dois níveis (proximal e distal). No primeiro nível foram incluídas as variáveis sociodemográficas e no segundo as variáveis comportamentais (Figura 1).

As análises levaram em consideração a seleção amostral por conglomerados (svyset) para ajuste das estimativas sem recurso à ponderação. No processo de modelagem (método foward) apenas as variáveis que apresentaram um valor de $\mathrm{p} \leq 0,20$ permaneceram no modelo. Após a seleção das variáveis independentes foi realizada a análise de multicolinearidade $(v i f<10)$. Para a avaliação do melhor modelo foi usada a análise dos valores de deviance. Os resultados foram apresentados em odds ratio (OR) e IC95\%. Foram consideradas variáveis associadas aquelas em que o valor de p foi inferior a 0,05 no modelo final. 


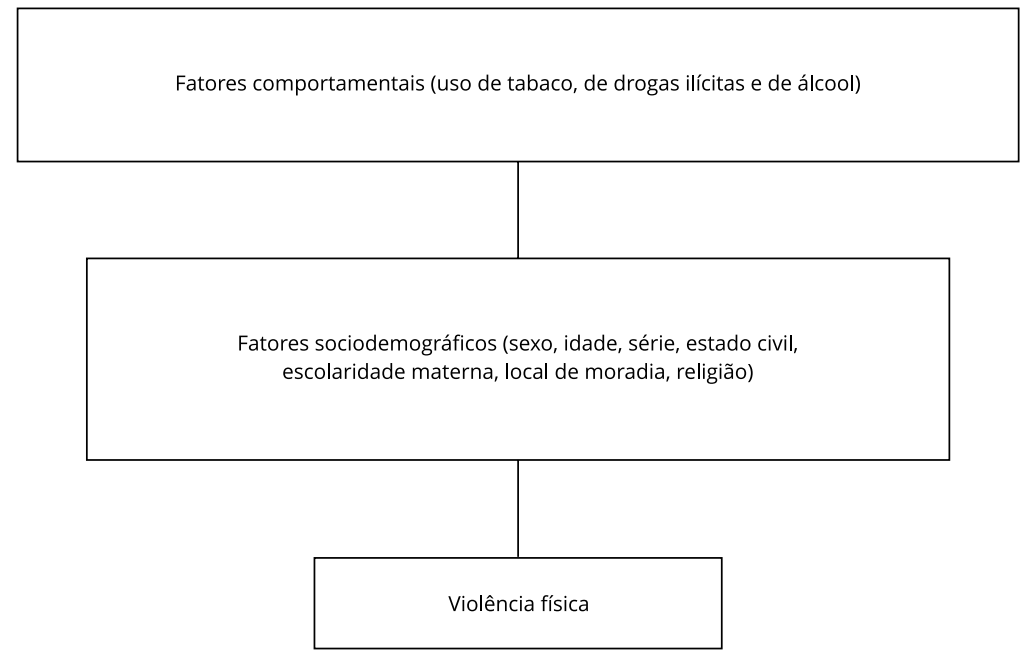

\section{Resultados}

Em 2006, 6.112 estudantes eram elegíveis para participar da coleta, e em 2011, 7.476 estavam aptos a participar da coleta. Considerando a quantidade de alunos presentes, o percentual de respostas válidas, respectivamente, em 2006 e 2011 foi de $98,6 \%$ e 96,2\%. Foram analisados somente os estudantes na faixa etária definida para este estudo (14 a 19 anos), na primeira coleta 4.207 adolescentes foram avaliados e na segunda (2011) 6.264. Observou-se em 2006 maior proporção entre rapazes de 17 a 19 anos, estudantes do 1o ano, solteiros, e uma maior proporção de moças entre os 14 e 16 anos, residentes na zona urbana, com escolaridade materna $\leq 8$ anos, no turno diurno e religião católica (Tabela 1).

Verificou-se que houve uma redução da prevalência do consumo de álcool, entre os anos de 2006 e 2011, dentre adolescentes que sofreram violência física ou que se envolveram em briga. Em relação a sofrer violência física, as moças apresentaram uma prevalência de consumo de álcool que variou de $14,4 \%$ em 2006 a 8,6\% em 2011. Os rapazes registraram uma prevalência maior, variando de 16,3\% a $11,4 \%$ nos respectivos anos. Ao avaliar o consumo de bebida alcoólica por adolescentes que se envolveram em brigas, a proporção de moças diminuiu de $25,1 \%$ para $15,3 \%$. Uma diminuição na prevalência também foi verificada nos rapazes, passando de 37,5\% em 2006 para 29,7\% em 2011 (Tabela 2).

Foram propostos quatro modelos, ajustados pelas variáveis sociodemográficas. Adolescentes que faziam uso de bebida alcoólica em 2006 tinham 1,63 (IC95\%: 1,31; 2,01) maior chance de sofrer violência física quando comparados a adolescentes que não consumiam álcool. Em 2011, essa chance aumentou para 1,84 (IC95\%: 1,44; 2,36). Em 2006, os estudantes que usavam drogas ilícitas registraram uma chance 2,12 maior de sofrer violência física e em 2011 este valor foi de 2,41 (IC95\%: 1,80; 3,22 ). Comportamento semelhante foi verificado ao avaliar o envolvimento em brigas. A chance de adolescentes que consumiam bebida alcoólica terem envolvimento em brigas aumentou de 1,96 (IC95\%: 1,64; 2,34) em 2006, para 2,11 (IC95\%: 1,78; 2,51) em 2011. O uso de drogas ilícitas também apresentou um aumento da chance de envolvimento em brigas após cinco anos (Tabela 3). 
Tabela 1

Características das amostras de estudantes do Ensino Médio (adolescentes, 14-19 anos), por sexo. Pernambuco, Brasil, 2006 e 2011.

\begin{tabular}{|c|c|c|c|c|c|c|}
\hline \multirow[t]{2}{*}{ Variável } & \multicolumn{3}{|c|}{$2006(n=4.207)$} & \multicolumn{3}{|c|}{$2011(n=6.264)$} \\
\hline & $\begin{array}{c}\text { Masculino } \\
\text { n (\%) }\end{array}$ & $\begin{array}{c}\text { Feminino } \\
\text { n (\%) }\end{array}$ & $\begin{array}{l}\text { Total } \\
\text { n (\%) }\end{array}$ & $\begin{array}{c}\text { Masculino } \\
\text { n (\%) }\end{array}$ & $\begin{array}{c}\text { Feminino } \\
\text { n (\%) }\end{array}$ & $\begin{array}{l}\text { Total } \\
\text { n (\%) }\end{array}$ \\
\hline \multicolumn{7}{|l|}{ Idade (anos) } \\
\hline $14-16$ & $598(35,4)$ & $1.165(46,4)$ & $1.766(42,0)$ & $1.109(43,9)$ & $1.919(51,3)$ & $3.028(48,3)$ \\
\hline $17-19$ & $1.089(64,6)$ & $1.346(53,6)$ & $2.441(58,0)$ & $1.416(56,1)$ & $1.820(48,7)$ & $3.236(51,7)$ \\
\hline \multicolumn{7}{|l|}{ Residência } \\
\hline Rural & $367(21,9)$ & $510(20,5)$ & $881(21,1)$ & $641(25,4)$ & $947(25,5)$ & $1588(25,5)$ \\
\hline Urbana & $1.311(78,1)$ & $1983(79,5)$ & $3.299(78,9)$ & $1.878(74,6)$ & $2.768(74,5)$ & $4.646(74,5)$ \\
\hline \multicolumn{7}{|c|}{ Escolaridade materna (anos) } \\
\hline$\leq 8$ & $1.086(69,4)$ & $1.771(74,5)$ & $2.865(72,5)$ & $1.324(60,5)$ & $2.167(67,6)$ & $3491(64,7)$ \\
\hline $9-11$ & $352(22,5)$ & $480(20,2)$ & $833(21,1)$ & $625(28,6)$ & $774(24,1)$ & $1.399(26,0)$ \\
\hline$\geq 12$ & $127(8,1)$ & $126(5,3)$ & $253(6,4)$ & $239(10,9)$ & $265(8,3)$ & $504(9,3)$ \\
\hline \multicolumn{7}{|l|}{ Série } \\
\hline 1ㅇa ano & $770(45,9)$ & $1.095(43,8)$ & $1.868(44,6)$ & $977(38,7)$ & $1.357(36,3)$ & $2.334(37,3)$ \\
\hline 2o ano & $524(31,2)$ & $808(32,3)$ & $1.336(31,9)$ & $804(31,9)$ & $1.216(32,5)$ & $2.020(32,3)$ \\
\hline 3o ano & $384(22,9)$ & $598(23,9)$ & $984(23,5)$ & $741(29,4)$ & $1.165(31,2)$ & $1.906(30,4)$ \\
\hline \multicolumn{7}{|l|}{ Turno } \\
\hline Diurno * & $908(53,9)$ & $1.506(60,1)$ & $2.415(57,5)$ & $1.726(69,0)$ & $2.721(73,5)$ & $4.447(71,7)$ \\
\hline Noturno & $778(46,1)$ & $1.002(39,9)$ & $1.788(42,5)$ & $775(31,0)$ & $979(26,5)$ & $1.754(28,3)$ \\
\hline \multicolumn{7}{|l|}{ Estado civil } \\
\hline Solteiro & $1.605(95,8)$ & $2.338(93,6)$ & $3.946(94,4)$ & $2.351(93,4)$ & $3.356(89,9)$ & $5.707(91,3)$ \\
\hline Casado & $28(1,7)$ & $113(4,5)$ & $143(3,4)$ & $63(2,5)$ & $227(6,1)$ & $290(4,5)$ \\
\hline Outro & $43(2,5)$ & $48(1,9)$ & $91(2,2)$ & $104(4,1)$ & $149(4,0)$ & $253(4,1)$ \\
\hline \multicolumn{7}{|l|}{ Cor da pele } \\
\hline Branca & $417(24,8)$ & $639(25,5)$ & $1.057(25,2)$ & $667(26,5)$ & $953(25,6)$ & $1.620(25,9)$ \\
\hline Preta & $884(52,7)$ & $1.194(47,7)$ & $2.085(49,7)$ & $386(15,3)$ & $404(10,8)$ & $790(12,7)$ \\
\hline Outras ** & $378(22,5)$ & $672(26,8)$ & $1.051(25,1)$ & $1463(58,2)$ & $2369(63,6)$ & $3.832(61,4)$ \\
\hline \multicolumn{7}{|l|}{ Religião } \\
\hline Não & $325(19,4)$ & $281(11,2)$ & $606(14,4)$ & $333(13,2)$ & $509(13,7)$ & $842(13,4)$ \\
\hline Católico & $926(55,1)$ & $1.541(61,6)$ & $2.474(59,1)$ & $1428(56,6)$ & $2075(55,6)$ & $3.503(56,0)$ \\
\hline Evangélico & $358(21,3)$ & $600(24,0)$ & $959(22,9)$ & $669(26,6)$ & $1016(27,2)$ & $1.685(27,0)$ \\
\hline Outros & $70(4,2)$ & $80(3,2)$ & $150(3,6)$ & $91(3,6)$ & $132(3,5)$ & $223(3,6)$ \\
\hline
\end{tabular}

* Estudantes dos turnos manhã e tarde;

** Pardo, amarelo e indígena.

\section{Discussão}

O presente estudo avaliou a associação de consumo de bebidas alcoólicas e uso de drogas ilícitas com violência física e envolvimento em brigas em adolescentes do Ensino Médio do Estado de Pernambuco, em 2006 e 2011. O consumo de bebida alcoólica e o uso de drogas ilícitas foram fatores de risco para sofrer violência física e para o envolvimento em brigas, e este risco aumentou ao longo dos cinco anos.

Neste estudo, quando comparada a prevalência do consumo de bebida alcoólica pelos adolescentes que sofreram violência física entre os anos 2006 e 2011 foi encontrada uma redução significativa. A implantação, em 2008, no Ensino Médio da rede pública do Estado de Pernambuco de várias escolas de referência 21, contribuiu como um fator protetor para violência física e comportamento de risco. O ensino integral em Pernambuco, assim como no Brasil, busca contemplar a realização das tarefas 
Tabela 2

Prevalência da violência segundo indicadores comportamentais entre estudantes do Ensino Médio (adolescentes, 14-19 anos), por sexo. Pernambuco, Brasil, 2006 e 2011.

\begin{tabular}{|c|c|c|c|c|c|c|c|c|c|c|c|c|}
\hline \multirow[t]{3}{*}{ Variáveis } & \multicolumn{6}{|c|}{ Feminino } & \multicolumn{6}{|c|}{ Masculino } \\
\hline & \multicolumn{2}{|c|}{$\begin{array}{c}2006 \\
(n=2.519)\end{array}$} & \multicolumn{2}{|c|}{$\begin{array}{c}2011 \\
(n=3.379)\end{array}$} & \multirow[t]{2}{*}{$\Delta \%$} & \multirow[t]{2}{*}{ Valor de $p$} & \multicolumn{2}{|c|}{$\begin{array}{c}2006 \\
(n=1.688)\end{array}$} & \multicolumn{2}{|c|}{$\begin{array}{c}2011 \\
(n=2.525)\end{array}$} & \multirow[t]{2}{*}{$\Delta \%$} & \multirow[t]{2}{*}{ Valor de p } \\
\hline & $\mathbf{n}$ & $\%(I C 95 \%)$ & $\mathbf{n}$ & $\%(I C 95 \%)$ & & & $\mathbf{n}$ & $\%($ IC95\%) & $\mathbf{n}$ & $\%$ (IC95\%) & & \\
\hline \multicolumn{13}{|c|}{ Violência física } \\
\hline \multicolumn{13}{|c|}{ Uso de álcool } \\
\hline Sim & 89 & $\begin{array}{c}14,4 \\
(0,12 ; 0,17)\end{array}$ & 203 & $\begin{array}{c}8,6 \\
(0,07 ; 0,10)\end{array}$ & $-40,3$ & $<0,01$ & 105 & $\begin{array}{c}16,3 \\
(0,13 ; 0,19)\end{array}$ & 184 & $\begin{array}{c}11,4 \\
(0,10 ; 0,13)\end{array}$ & $-30,1$ & $<0,01$ \\
\hline \multicolumn{13}{|c|}{$\begin{array}{l}\text { Uso do de drogas } \\
\text { ilícitas }\end{array}$} \\
\hline Sim & 26 & $\begin{array}{c}21,9 \\
(0,14 ; 0,30)\end{array}$ & 35 & $\begin{array}{c}19,7 \\
(0,13 ; 0,26)\end{array}$ & $-10,0$ & 0,66 & 43 & $\begin{array}{c}25,6 \\
(0,19 ; 0,32)\end{array}$ & 50 & $\begin{array}{c}21,5 \\
(0,16 ; 0,27)\end{array}$ & $-16,0$ & 0,33 \\
\hline \multicolumn{13}{|c|}{$\begin{array}{l}\text { Envolvimento em } \\
\text { brigas }\end{array}$} \\
\hline \multicolumn{13}{|c|}{ Uso de álcool } \\
\hline Sim & 155 & $\begin{array}{c}25,1 \\
(0,22 ; 0,29)\end{array}$ & 361 & $\begin{array}{c}15,3 \\
(0,14 ; 0,17)\end{array}$ & $-61,0$ & $<0,01$ & 242 & $\begin{array}{c}37,5 \\
(0,33 ; 0,42)\end{array}$ & 480 & $\begin{array}{c}29,7 \\
(0,27 ; 0,32)\end{array}$ & $-20,8$ & $<0,01$ \\
\hline \multicolumn{13}{|c|}{$\begin{array}{l}\text { Uso do de drogas } \\
\text { ilícitas }\end{array}$} \\
\hline Sim & 48 & $\begin{array}{c}40,3 \\
(0,31 ; 0,49)\end{array}$ & 62 & $\begin{array}{c}34,6 \\
(0,27 ; 0,42)\end{array}$ & $-14,1$ & 0,33 & 85 & $\begin{array}{c}50,3 \\
(0,42 ; 0,58)\end{array}$ & 116 & $\begin{array}{c}50,0 \\
(0,43 ; 0,57)\end{array}$ & $-0,6$ & 0,96 \\
\hline
\end{tabular}

IC95\%: intervalo de 95\% de confiança.

escolares, acompanhamento dos estudos, oferta de lanches e refeições ao aluno, além das atividades que compõem a proposta pedagógica escolar deste tipo de educação 22 . A estrutura da escola também é diferente e oferece espaços de convivência, todas estas características propiciam ao adolescente ter suas potencialidades desenvolvidas, sendo mais vantajoso do que ficar horas de forma ociosa sem a supervisão adequada em casa. Ademais, aumenta o vínculo com a escola e amigos, desenvolvendo suas relações interpessoais.

A prevalência de adolescentes que sofreram violência foi similar à de outros estudos 12,23. Em relação ao envolvimento em brigas, a prevalência foi superior à encontrada em adolescentes do 9o ano do Ensino Fundamental 24 e inferior quando comparada a um estudo com a mesma faixa etária 25 . A prevalência de estudantes que sofreram violência física e consumiam bebidas alcoólicas variou de $8 \%$ a 37\%. No Estado de Mato Grosso, dos adolescentes que consumiram álcool no último mês, 35\% se envolveram em brigas 26, já na Malásia, esta porcentagem foi ainda maior, onde 40,8\% dos adolescentes que consumiram álcool se envolveram em briga 27.

O consumo de álcool e o uso de drogas ilícitas aumentaram a chance do estudante sofrer violência física e ter se envolvido em brigas, assim como em outros estudos 11,13,25,28. Sugere-se que o efeito psicoativo dessas substâncias leva a um aumento do comportamento agressivo, perda do senso crítico e consequentemente um maior envolvimento em situações violentas 27 . Os efeitos do álcool podem causar distorção cognitiva e de percepção, déficit de atenção, julgamento errado de uma situação e mudanças neuroquímicas, originando ou estimulando comportamentos violentos. $\mathrm{O}$ uso crônico pode colaborar com agressões devido à privação de sono, abstinência e prejuízo de funcionamento neuropsicológico 29.

Alguns esforços vêm sendo feitos pelo governo no combate ao uso de álcool e de drogas, mas eles ainda parecem ser incipientes. Apesar da criação em 2006 da Lei no 11.343/2006, que instituiu o Sistema Nacional de Políticas Públicas sobre Drogas (SISNAD), o estudo mostrou uma associação entre o 
Tabela 3

Associação entre o uso de álcool e o uso de drogas ilícitas com vitimização por violência física e envolvimento em brigas em entre estudantes do Ensino Médio (adolescentes, 14-19 anos). Pernambuco, Brasil, 2006 e 2011.

\begin{tabular}{|c|c|c|c|c|}
\hline \multirow[t]{2}{*}{ Variáveis } & \multicolumn{2}{|c|}{2006} & \multicolumn{2}{|c|}{2011} \\
\hline & $\begin{array}{l}\text { OR bruto } \\
\text { (IC95\%) }\end{array}$ & $\begin{array}{l}\text { OR ajustado * } \\
\text { (IC95\%) }\end{array}$ & $\begin{array}{l}\text { OR bruto } \\
\text { (IC95\%) }\end{array}$ & $\begin{array}{l}\text { OR ajustado ** } \\
\text { (IC95\%) }\end{array}$ \\
\hline \multicolumn{5}{|c|}{ Violência física } \\
\hline \multicolumn{5}{|c|}{ Uso de álcool } \\
\hline Não & 1,00 & 1,00 & 1,00 & 1,00 \\
\hline \multirow[t]{2}{*}{ Sim } & 1,92 & 1,63 & 2,19 & 1,84 \\
\hline & $(1,56 ; 2,36)$ & $(1,31 ; 2,01)$ & $(1,72 ; 2,81)$ & $(1,44 ; 2,36)$ \\
\hline \multicolumn{5}{|c|}{ Uso de drogas ilícitas } \\
\hline Não & 1,00 & 1,00 & 1,00 & 1,00 \\
\hline \multirow[t]{2}{*}{ Sim } & 2,91 & 2,12 & 3,47 & 2,41 \\
\hline & $(2,21 ; 3,84)$ & $(1,59 ; 2,83)$ & $(2,63 ; 4,58)$ & $(1,80 ; 3,22)$ \\
\hline \multicolumn{5}{|c|}{ Envolvimento em brigas } \\
\hline \multicolumn{5}{|c|}{ Uso de álcool } \\
\hline Não & 1,00 & 1,00 & 1,00 & 1,00 \\
\hline \multirow[t]{2}{*}{ Sim } & 2,30 & 1,96 & 2,40 & 2,11 \\
\hline & $(1,96 ; 2,70)$ & $(1,64 ; 2,34)$ & $(2,06 ; 2,80)$ & $(1,78 ; 2,51)$ \\
\hline \multicolumn{5}{|c|}{ Uso de drogas ilícitas } \\
\hline Não & 1,00 & 1,00 & 1,00 & 1,00 \\
\hline \multirow[t]{2}{*}{ Sim } & 3,58 & 2,25 & 4,25 & 2,56 \\
\hline & $(2,80 ; 4,57)$ & $(1,73 ; 2,93)$ & $(3,45 ; 5,23)$ & $(2,07 ; 3,23)$ \\
\hline
\end{tabular}

OR: odds ratio; IC95\%: intervalo de 95\% de confiança.

* Violência física: ajustado por fumo, religião e local de moradia; envolvimento em brigas: ajustado por fumo, local de moradia, série e idade;

** Violência física: ajustado por fumo, estado civil e série; envolvimento em brigas: ajustado por fumo, sexo, série, estado civil e escolaridade materna.

uso de drogas e ter sofrido violência física em ambos os levantamentos, mostrando um aumento na magnitude desta associação no ano de 2011, em comparação com o ano de 2006.

Os resultados do presente estudo devem ser interpretados com cautela. Este é um estudo de painel e, portanto, não permite inferir sobre causalidade na interpretação dos resultados. Os fatores foram selecionados na literatura especializada, mas as interpretações de causa e efeito devem ser feitas com cuidado. É possível que a prevalência do consumo de bebida alcoólica, do uso de drogas ilícitas e sofrer violência física seja ainda maior do que a apresentada, pois embora a natureza da pesquisa e a segurança do anonimato nas respostas tenham sido explicadas, os adolescentes podem ter ficado inseguros ou com vergonha de admitir comportamentos socialmente inaceitáveis.

Em contrapartida, alguns pontos fortes desta pesquisa devem ser destacados. Ela tem elevada validade externa, apresentando resultados representativos dessa população, que podem ser cuidadosamente extrapolados em nível nacional e para outros países em desenvolvimento. Além disso, esses resultados podem ser úteis em futuras estratégias de saúde pública para adolescentes brasileiros, uma vez que descreve uma elevada prevalência do consumo de álcool, uso de drogas ilícitas e violência sofrida pelos adolescentes. 


\section{Conclusão}

Conclui-se que apesar do consumo de álcool pelos estudantes que sofreram violência física ou que se envolveram em briga diminuir em cinco anos, o risco daqueles que consumiram bebida alcoólica ou usaram drogas ilícitas terem sofrido violência física aumentou ao longo de cinco anos. Os mecanismos para essas alterações precisam ser melhor investigados para um correto planejamento, implementação e avaliação de políticas de educação e saúde para a redução de comportamentos de violência física em adolescentes.

\section{Colaboradores}

D. R. Queiroz contribuiu com a aquisição, análise e interpretação dos dados, redação e revisão crítica do manuscrito. M. V. G. Barros, J. A. Aguilar, F. C. Soares, R. M. Tassitano e J. Bezerra contribuíram com o desenho do estudo, análise e interpretação dos dados, redação e revisão crítica do manuscrito. L. M. P. Silva contribuiu com a concepção e desenho do estudo, aquisição, análise e interpretação dos dados, redação e revisão crítica do manuscrito. Todos os autores aprovaram o texto final e são responsáveis por todos os aspectos do trabalho.

\section{Informações adicionais}

ORCID: Daniel da Rocha Queiroz (0000-00029901-4677); Mauro Virgílio G. de Barros (00000003-3165-0965); Javiera Alarcón Aguilar (00000003-4267-046X); Fernanda Cunha Soares (0000-0001-6465-3164); Rafael de Miranda Tassitano (0000-0002-2713-8670); Jorge Bezerra (00000002-9935-4508); Lygia Maria Pereira da Silva (0000-0003-4512-4990).

\section{Agradecimentos}

Agradecimento a Coordenação de Aperfeiçoamento de Pessoal de Nível Superior (CAPES), ao Conselho Nacional de Desenvolvimento Científico e Tecnológico $(\mathrm{CNPq})$ e à Fundação de Amparo a Ciência e Tecnologia de Pernambuco (FACEPE).

\section{Referências}

1. Minayo MCS. Violência social sob a perspectiva da saúde pública. Cad Saúde Pública 1994; 10 Suppl 1:7-18.

2. Daro D. A public health approach to prevention: what will it take? Trauma Violence Abuse 2016; 17:420-1.

3. Moffitt TE, Tank K-G. Childhood exposure to violence and lifelong health: clinical intervention science and stress-biology research join forces. Dev Psychopathol 2013; 25(4 Pt 2):1619-34.

4. Andrade SSCA, Yokota RTC, Sá NNB, Silva MMA, Araújo WN, Mascarenhas MDM, et al. Relação entre violência física, consumo de álcool e outras drogas e bullying entre adolescentes escolares brasileiros. Cad Saúde Pública 2012; 28:1725-36.

5. Otwombe KN, Dietrich J, Sikkema KJ, Coetzee J, Hopkins KL, Laher F, et al. Exposure to and experiences of violence among adolescents in lower socio-economic groups in Johannesburg, South Africa. BMC Public Health 2015; 15:450.

6. Heinze JE, Stoddard SA, Aiyer SM, Eisman AB, Zimmerman MA. Exposure to violence during adolescence as a predictor of perceived stress trajectories in emerging adulthood. J Appl Dev Psychol 2017; 49:31-8.

7. Miller E. Prevention of and interventions for dating and sexual violence in adolescence. Pediatr Clin North Am 2017; 64:423-34.

8. Pinto IV, Barufaldi LA, Campos MO, Malta DC, Souto RMCV, Freitas MG, et al. Tendências de situações de violência vivenciadas por adolescentes brasileiros: Pesquisa Nacional de Saúde do Escolar 2009, 2012 e 2015. Rev Bras Epidemiol 2018; 21 Suppl 1:e180014.

9. Waiselsz J. Mapa da violência 2012: crianças e adolescentes do Brasil. Rio de Janeiro: FLACSO Brasil; 2012. 
10. Institute of Medicine; National Research Council. The science of adolescent risk-taking: workshop report. Washington DC: National Academies Press; 2011.

11. Beserra MA, Carlos DM, Leitão MNDC, Ferriani MDGC. Prevalence of school violence and use of alcohol and other drugs in adolescents. Rev Latinoam Enferm 2019; 27:e3110.

12. Carvalho AP, Silva TCD, Valença PAM, Ferreira Santos CDFB, Colares V, Menezes VA. Consumo de álcool e violência física entre adolescentes: quem é o preditor? Ciênc Saúde Colet 2017; 22:4013-20.

13. Kobulsky JM, Minnes S, Min MO, Singer MI. Violence exposure and early substance use in high-risk adolescents. J Soc Work Pract Addict 2016; 16:46-71.

14. Minamisava R, Nouer SS, Morais Neto OL, Melo LK, Andrade ALSS. Spatial clusters of violent deaths in a newly urbanized region of Brazil: highlighting the social disparities. Int J Health Geogr 2009; 8:66.

15. Assis SG, Avanci JQ, Pesce RP, Pires TO, Gomes DL. Notificações de violência doméstica, sexual e outras violências contra crianças no Brasil. Ciênc Saúde Colet 2012; 17:2305-17.

16. Condessa LA, Soares CA, Mielke GI, Malta DC, Caiaffa WT. Prevalência de adolescentes fisicamente ativos nas capitais brasileiras: Pesquisa Nacional de Saúde do Escolar 2012 e 2015. Rev Bras Epidemiol 2018; 21 Suppl $1: \mathrm{e} 180012$

17. Barreira AK, Lima MLC, Avanci JQ. Coocorrência de violência física e psicológica entre adolescentes namorados do Recife, Brasil: prevalência e fatores associados. Ciênc Saúde Colet 2013; 18:233-43.

18. Medronho R. Epidemiologia. São Paulo: Atheneu; 2006.

19. Carvalho PD, Barros MVG, Santos CM, Melo EN, Oliveira NKR, Lima RA. The prevalence of and factors associated with negative mental health indicators in adolescents attending high school in the Brazilian state of Pernambuco. Rev Bras Saúde Matern Infant 2011; 11:22738.
20. Bronfenbrenner U. Toward and experimental ecology of human development. Am Psychol 1977; 32:513-31.

21. Estado de Pernambuco. Lei Complementar no 125 , de 10 de julho de 2008. Diário Oficial do Estado de Pernambuco 2008; 11 jul.

22. Gadotti M. Educação integral no Brasil: inovações em processo. São Paulo: Editora e Livraria Instituto Paulo Freire; 2009.

23. De Long SM, Powers KA, Pence BW, Maman S, Dunkle KL, Selin A, et al. Longitudinal trajectories of physical intimate partner violence among adolescent girls in rural South Africa: findings from HPTN 068. J Adolesc Health 2020; 67:69-75.

24. Malta DC, Souza ER, Silva MMA, Silva CS, Andreazzi MAR, Crespo C, et al. Vivência de violência entre escolares brasileiros: resultados da Pesquisa Nacional de Saúde do Escolar (PeNSE). Ciênc Saúde Colet 2010; 15:3053-63.

25. Silva RA, Jansen K, Godoy RV, Souza LDM, Horta BL, Pinheiro RT. Prevalência e fatores associados a porte de arma e envolvimento em agressão física entre adolescentes de 15 a 18 anos: estudo de base populacional. Cad Saúde Pública 2009; 25:2737-45.

26. Castro ML, Cunha SS, Souza DPO. Comportamento de violência e fatores associados entre estudantes de Barra do Garças, MT. Rev Saúde Pública 2011; 45:1054-61.

27. Mat Hussin SF, Abd Aziz NS, Hasim H, Sahril N. Prevalence and factors associated with physical fighting among Malaysian adolescents. Asia Pac J Public Health 2014; $26(5$ Suppl):108S-15.

28. Faria CS, Martins CBG. Violência entre adolescentes escolares: condições de vulnerabilidades. Enferm Glob 2016; 42:157-98.

29. Spear LP. Effects of adolescent alcohol consumption on the brain and behaviour. Nat Rev Neurosci 2018; 19:197-214. 
Abstract

The article aimed to estimate prevalence and analyze the association between alcohol and illicit drug consumption and indicators of physical violence in adolescents. A repeated cross-sectional study was performed in public middle-school students in the State of Pernambuco, Brazil (2006, $n=4,207 ; 2011, n=6,264)$. Data were obtained via a questionnaire. Indicators of physical violence were: victimization by physical violence and involvement in fights. The independent variables were alcohol and illicit drug consumption. Binary logistic regression was used to verify the association between alcohol and drug consumption and victimization from physical violence. Prevalence of alcohol consumption in adolescents that suffered physical violence decreased in five years, but no change appeared in the use of illicit drugs. The behavioral variables were associated with different indicators of violence, generally presenting an upward trend. In 2006, adolescents that used illicit drugs showed 2.12 higher odds of suffering physical violence, and in 2011 this value was 2.41 (95\% CI: 1.80; 3.22). The odds of adolescents that consumed alcoholic beverages being involved in fights increased from 1.96 in 2006 (95\% CI: 1.64; $2.34)$ to 2.11 in 2011 (95\%CI: 1.78; 2.51). Alcohol consumption by adolescents that suffered physical violence decreased in five years, but the risk of adolescents that consumed alcohol or used illicit drugs and suffered physical violence increased over the course of five years.

Alcohol Drinking; Ilicit Drugs; Adolescent Health; Violence

\section{Resumen}

El objetivo fue estimar la prevalencia y analizar la asociación del consumo de alcohol y drogas ilícitas con indicadores de violencia física en adolescentes. Se realizó un estudio transversal, repetido con estudiantes de enseñanza media en escuelas de la red pública en Pernambuco, Brasil (2006, $n=4.207$; $2011, n=6.264$ ). Los datos se obtuvieron mediante cuestionario. Los indicadores de violencia física fueron: victimización por violencia física; implicación en peleas. Las variables independientes fueron consumo de alcohol y uso de drogas ilícitas. La regresión logística binaria fue utilizada para verificar la asociación entre consumo de alcohol y drogas y haber sufrido violencia física. La prevalencia del consumo de alcohol en adolescentes que sufrieron violencia física se redujo en cinco años, a pesar de que no se constató ninguna alteración en el uso de drogas ilícitas. Las variables comportamentales estuvieron asociadas a los diferentes indicadores de violencia, presentando en general una tendencia de aumento. En 2006, adolescentes que usaban drogas ilícitas presentaron una oportunidad 2,12 mayor de sufrir violencia física y en 2011 ese valor fue de 2,41 (IC95\%: 1,80; 3,22). La oportunidad de adolescentes que consumian bebidas alcohólicas de que tuvieran implicación en peleas aumentó de 1,96 (IC95\%: 1,64; 2,34) en 2006, a 2,11 (IC95\% 1,78; 2,51) en 2011. El consumo de alcohol por parte de los adolescentes que sufrieron violencia física disminuyó en cinco años, no obstante, el riesgo de los adolescentes que consumieron bebidas alcohólica o consumieron drogas ilícitas sufrieran violencia física aumentó a lo largo de cinco años.

Consumo de Bebidas Alcohólicas; Drogas Ilícitas; Salud del Adolescente; Violencia
Recebido em 19/Mar/2020

Versão final reapresentada em 20/Jul/2020

Aprovado em 31/Ago/2020 